\title{
One-dimensional nested maximin designs
}

\author{
Edwin R. van Dam • Bart Husslage • Dick den Hertog
}

Received: 24 August 2006 / Accepted: 23 April 2009 / Published online: 8 May 2009

(C) The Author(s) 2009. This article is published with open access at Springerlink.com

\begin{abstract}
The design of computer experiments is an important step in black-box evaluation and optimization processes. When dealing with multiple black-box functions the need often arises to construct designs for all black boxes jointly, instead of individually. These so-called nested designs are particularly useful as training and test sets for fitting and validating metamodels, respectively. Furthermore, nested designs can be used to deal with linking parameters and sequential evaluations. In this paper, we introduce one-dimensional nested maximin designs. We show how to nest two designs optimally and develop a heuristic to nest three and four designs. These nested maximin designs can be downloaded from the website http:// www.spacefillingdesigns.nl. Furthermore, it is proven that the loss in space-fillingness, with respect to traditional maximin designs, is at most 14.64 and $19.21 \%$, when nesting two and three designs, respectively.
\end{abstract}

Keywords Computer simulation - Global optimization - Linking parameter · Maximin design · Mixed integer linear programming · Packing problem $\cdot$ Space-filling · Training and test set

The research of E. R. van Dam has been made possible by a fellowship of the Royal Netherlands Academy of Arts and Sciences.

The research of B. G. M. Husslage has been financially supported by the Samenwerkings Orgaan Brabantse Universiteiten (SOBU).

E. R. van Dam $(\varangle) \cdot$ B. Husslage $\cdot$ D. den Hertog

Department of Econometrics and Operations Research, Tilburg University, P.O. Box 90153, 5000 LE

Tilburg, The Netherlands

e-mail: Edwin.vanDam@uvt.nl

B. Husslage

e-mail: Husslage@casema.nl

D. den Hertog

e-mail: D.denHertog@uvt.nl 


\section{Introduction}

Maximin designs play an important role in the area of (deterministic) black-box evaluation and optimization. By nature, a black-box function is not given explicitly, however, we may perform function evaluations. Based on these evaluations an approximation model for the black box can be constructed, which leads to more insight in the black box and which opens the way for optimization techniques. Unfortunately, function evaluations often constitute time-consuming computer simulations, thereby limiting the number of evaluations performed. A proper design of computer experiments then becomes vitally important, see e.g., Sacks et al. (1989), Jones et al. (1998), Booker et al. (1999), Myers (1999), den Hertog and Stehouwer (2002), and Kleijnen and van Beers (2004).

We will use the term design to denote the set of points that will be evaluated. Such a design should at least be space-filling in some sense to provide information about the entire black-box domain. Several space-filling measures, like maximin, minimax, IMSE, and maximum entropy, are used in the literature; see e.g., Montgomery (1984), Sacks et al. (1989), and Morris and Mitchell (1995). A good survey of these criteria can be found in Santner et al. (2003), in which it is also shown that maximum entropy and distance-based criteria are preferable when conducting computer experiments. We will therefore consider the maximin criterion, which maximizes the minimal distance among all pairs of points, in this paper. Maranas et al. (1995) and Nurmela and Östergård (1997, 1999) among others, consider maximin designs in two dimensions, whereas Gensane (2004) considers maximin designs in three dimensions. Finding the maximin design is in fact an optimization problem with many local optima. Global optimization techniques are used in, e.g., Pintér (2001), Stortelder et al. (2001), and Pintér et al. (2008), to solve such problems. A collection of other maximin problems and solution techniques can be found in Du and Pardalos (1995). For maximin Latin hypercube designs, the reader is referred to van Dam et al. $(2007,2009)$ and Husslage et al. (2008).

In real-life problems there is often a need for nested designs. This type of design consists of two separate designs, with the requirement that one design is a subset of the other design. Such nested designs can be found by mixed integer linear programming, however, this may be computationally expensive, or even impossible, in particular for larger problem instances. This paper shows how to construct one-dimensional nested maximin designs. Furthermore, it is proven that the loss in space-fillingness, with respect to traditional maximin designs, is at most 14.64 and $19.21 \%$, when nesting two and three designs, respectively. There are three main reasons for nesting maximin designs: training and test sets, linking parameters, and sequential evaluations.

To start with the first, consider the problem of fitting and validating a particular metamodel. First, this approximation model is fitted to the obtained data, i.e., the responses obtained when evaluating the design points in the training set. Then, a new set of design points, i.e., the test set, is evaluated and the obtained responses are compared to the response values predicted by the metamodel. If the differences between the predicted and the actual response values are small the metamodel is said to be valid. Training and test sets are often used in, for example, neural networks. See also Cherkassky and Mulier (1998) for a more detailed description of the use of training and test sets, and Hurrion (1997) and Su et al. (2005) for several interesting neural network applications. Since a metamodel should be a global approximation model, i.e., it should be valid for the entire feasible region, the evaluation points, in both the training set and the test set, should cover the entire region. Moreover, the evaluation points in the test set should not lie too close to the evaluation points in the training set, i.e., the total set of evaluation points should be space-filling. Note that this is accomplished by nesting two 
designs, say, $X_{1}$ and $X_{2}$, with respect to, for example, the maximin criterion. The sets $X_{1}$ and $X_{2} \backslash X_{1}$ can then be used as the training set and the test set, respectively.

Another reason for using nested designs is caused by linking parameters. Consider a product that consists of two components, each of them represented by a black-box function. In practice it often occurs that the functions have an input parameter in common, also called a linking parameter, see Husslage et al. (2003). Evaluating such a linking parameter at the same setting in both functions (i.e., component-wise) leads to an evaluation of the product. Not only do product evaluations provide a better understanding of the product, they are also very useful in the product optimization process. Another reason for using the same settings for (linking) parameters is due to physical restrictions on the simulation tools. Setting the parameters for computer experiments can be a time-consuming job in practice, since characteristics, like shape and structure, have to be redefined for every new experiment. Therefore, it is preferable to use the same settings as much as possible. By constructing nested designs, these common settings for the parameters can be determined.

Sequential evaluations are a third reason for using nested designs. In practice, it is common that after evaluating an initial set of design points, extra evaluations are needed. As an example, suppose we construct an approximation model for a black-box function based on $n_{1}$ function evaluations. However, after validating the obtained model it turns out that an extra set of, say, $n_{2}-n_{1}$ function evaluations is needed to properly fit the current model. We then face the problem of constructing a design on $n_{2}$ points, given the initial design on $n_{1}$ points. It would be better to anticipate on the possibility of extra evaluations. This can be accomplished by constructing the two designs (on $n_{1}$ and $n_{2}$ points) at once, hence, by constructing a nested design.

We will now give a more strict formulation of our problem. Let there be $m \in \mathbb{N}$ nested sets (or designs) $X_{1} \subseteq X_{2} \subseteq \cdots \subseteq X_{m}$ and index sets $I_{1} \subseteq I_{2} \subseteq \cdots \subseteq I_{m}=\left\{1, \ldots, n_{m}\right\}$, where $X_{i}=\left\{x_{j} \mid j \in I_{i}\right\}$ and $\left|I_{i}\right|=n_{i}, i=1, \ldots, m$. Thus $I_{i}$ tells us which $x_{j}$ are contained in set $X_{i}$, and the $X_{i}$ 's define the nested design. We assume without loss of generality that all points $x_{j} \in[0,1]$. Note that when we consider a set $X_{i}$ independently, a space-filling distribution of the $x_{j}$ over the interval $[0,1]$ is obtained by spreading the points equidistantly over the interval, resulting in a minimal distance of $\frac{1}{n_{i}-1}$ among the points. Our aim is to determine $x_{j}$ and $I_{i}$ such that every set $X_{i}$ is as much as possible space-filling with respect to the maximin criterion. To this end we define $d_{i}$ as the minimal scaled distance among all points in the set $X_{i}$, i.e., $d_{i}=\min _{j, k \in I_{i}, j \neq k}\left(n_{i}-1\right)\left|x_{j}-x_{k}\right|$ for all $i$. Then, we have to maximize $d=\min _{i} d_{i}$ over all $I_{1} \subseteq I_{2} \subseteq \cdots \subseteq I_{m}$, with $\left|I_{i}\right|=n_{i}$, and $x_{j} \in[0,1]$, i.e., we get the following mathematical program:

$$
\begin{array}{lll}
\max & \min _{\substack{j, k \in I_{i} ; j \neq k \\
i=1, \ldots, m}}\left(n_{i}-1\right)\left|x_{j}-x_{k}\right| & \\
& & \\
\text { s.t. } & I_{1} \subseteq I_{2} \subseteq \cdots \subseteq I_{m}=\left\{1, \ldots, n_{m}\right\} & \\
& \left|I_{i}\right|=n_{i}, & i=1, \ldots, m-1 \\
& 0 \leq x_{j} \leq 1, & j \in I_{m} .
\end{array}
$$

This will yield the maximin distance $d$ and a corresponding nested maximin design in terms of the $I_{i}$ 's and $x_{j}$ 's.

Note that above formulation of the nested maximin design problem is again a nonlinear optimization problem for which global optimization techniques could be used. In this paper, however, we derive analytical solutions for the case of $m=2$, and for $m=3$ and $m=4$ we derive lower bounds for the maximin distance $d$ and propose a mixed integer linear 
programming formulation to obtain exact solutions. Unfortunately, when the number of design points is large, the corresponding mixed integer linear programming problem cannot be solved (within a reasonable amount of time). Therefore, we propose a heuristic to obtain approximate solutions.

This paper is organized as follows. In Sect. 2, we derive an exact formula for the maximin distance of two nested sets. This derivation also shows how to construct the corresponding nested maximin designs. In Sect. 3, we continue with three nested sets, for which we prove a lower bound on the maximin distance and develop a heuristic to construct good nested designs. Section 4 shows that the heuristic for three nested sets can also be used to construct good nested designs for four nested sets. Furthermore, in this section we prove a lower bound on the maximin distance for all $m \in \mathbb{N}$ that satisfy the restriction $n_{m}<2 n_{1}$. Finally, Sect. 5 provides the conclusions and some topics for further research.

\section{Two nested sets}

We first discuss the case of two nested sets, i.e., $m=2$. Note that this case is of particular interest when using the sets $X_{1}$ and $X_{2} \backslash X_{1}$ as a training set and a test set, respectively. In Sect. 2.1, we start with the general problem formulation and show how to nest two sets optimally. Furthermore, in this section we derive a formula for the maximin distance and prove a tight lower bound on this distance. In Sect. 2.2, we introduce the notion of dominance and discuss the trade-off between $d_{1}$ and $d_{2}$.

\subsection{Maximin distance}

The general problem for two nested sets can be formalized as the following mathematical program:

$$
\begin{array}{lc}
\max & \min _{\substack{j, k \in I_{i} \\
i=1,2 ; j \neq k}}\left(n_{i}-1\right)\left|x_{j}-x_{k}\right| \\
\text { s.t. } & I_{1} \subseteq I_{2}=\left\{1, \ldots, n_{2}\right\} \\
& \left|I_{1}\right|=n_{1} \\
& 0 \leq x_{j} \leq 1, \quad j \in I_{2} .
\end{array}
$$

To obtain a feasible solution that maximizes the objective function in (2), we may choose without loss of generality $x_{1}=0, x_{n_{2}}=1, x_{i}<x_{i+1}, 1 \in I_{1}$, and $n_{2} \in I_{1}$. For a given $I_{1}$, containing the indices, say, $1=a_{1}<a_{2}<\cdots<a_{n_{1}}=n_{2}$ we introduce the sequence $v=\left(v_{1}, \ldots, v_{n_{1}-1}\right)$ given by $v_{i}=a_{i+1}-a_{i}$. Thus $v_{i}-1$ gives the number of additional points of $X_{2}$ between the $i$-th and $(i+1)$-st point of $X_{1}$. It is clear that the set of possible $I_{1}$ is in one-one correspondence to the set of positive integral sequences $v$, summing to $n_{2}-1$. Now the approach to solve problem (2) is to first fix $I_{1}$, and its corresponding $a=\left(a_{1}, \ldots, a_{n_{1}}\right)$ and $v$, and obtain an expression for the maximal distance $\delta_{v}$, subject to the remaining constraints, and then to maximize $\delta_{v}$ over all $v$. It turns out that finding $\delta_{v}$ is rather simple.

Lemma 1 For fixed $I_{1}$, and corresponding $a$, $v$, the optimal value $\delta_{v}$ equals

$$
\left(\sum_{i=1}^{n_{1}-1} \max \left\{\frac{v_{i}}{n_{2}-1}, \frac{1}{n_{1}-1}\right\}\right)^{-1} .
$$


Proof Fix $a, v$, and let $\delta_{v}$ be the corresponding maximal distance. Since $x_{i+1}-x_{i} \geq \frac{\delta_{v}}{n_{2}-1}$ for all $i$, we have that $x_{a_{i+1}}-x_{a_{i}} \geq v_{i} \frac{\delta_{v}}{n_{2}-1}$. We also have that $x_{a_{i+1}}-x_{a_{i}} \geq \frac{\delta_{v}}{n_{1}-1}$, hence

$$
x_{a_{i+1}}-x_{a_{i}} \geq \max \left\{v_{i} \frac{\delta_{v}}{n_{2}-1}, \frac{\delta_{v}}{n_{1}-1}\right\} .
$$

From this we find that

$$
1=x_{a_{n_{1}}}-x_{a_{1}} \geq \delta_{v} \sum_{i=1}^{n_{1}-1} \max \left\{\frac{v_{i}}{n_{2}-1}, \frac{1}{n_{1}-1}\right\},
$$

which shows that the stated expression for $\delta_{v}$ is an upper bound. It is clear from the above that, and how, this upper bound can be attained, which proves the lemma.

We now have to maximize $\delta_{v}$ over all appropriate sequences $v$. For ease of notation define $c_{2}=\frac{n_{2}-1}{n_{1}-1}$.

Proposition 1 Let $2 \leq n_{1} \leq n_{2}$. The maximin distance in (2) is given by

$$
d=\frac{1}{1+\left\lfloor c_{2}\right\rfloor+\left\lceil c_{2}\right\rceil-c_{2}-\left\lfloor c_{2}\right\rfloor\left\lceil c_{2}\right\rceil \frac{1}{c_{2}}} .
$$

Proof As mentioned before, we have to maximize $\delta_{v}$, which is equivalent to minimizing

$$
\sum_{i=1}^{n_{1}-1} \max \left\{\frac{v_{i}}{n_{2}-1}, \frac{1}{n_{1}-1}\right\}
$$

over all integer-valued $v$, such that $\sum_{i=1}^{n_{1}-1} v_{i}=n_{2}-1$.

We claim that it is optimal to let $v$ only take values $\left\lfloor c_{2}\right\rfloor$ and $\left\lceil c_{2}\right\rceil$. This is clearly true if $n_{2}-1$ is a multiple of $n_{1}-1$, since in that case picking a larger value than $c_{2}$ for any of the $v_{i}$ will increase the objective function. Therefore, assume now that $n_{2}-1$ is not a multiple of $n_{1}-1$. To prove our claim, first assume that $v_{i} \leq\left\lfloor c_{2}\right\rfloor-1$ for some $i$. Let $j$ be such that $v_{j} \geq\left\lceil c_{2}\right\rceil$ (such a $j$ exists). Then by adding 1 to $v_{i}$, and subtracting 1 from $v_{j}$, we obtain $v^{\prime}$ for which the objective function is strictly smaller than for $v$. This follows from the inequality

$$
\begin{array}{r}
\max \left\{\frac{v_{i}}{n_{2}-1}, \frac{1}{n_{1}-1}\right\}+\max \left\{\frac{v_{j}}{n_{2}-1}, \frac{1}{n_{1}-1}\right\} \\
>\max \left\{\frac{v_{i}+1}{n_{2}-1}, \frac{1}{n_{1}-1}\right\}+\max \left\{\frac{v_{j}-1}{n_{2}-1}, \frac{1}{n_{1}-1}\right\},
\end{array}
$$

which is easily checked to be true. Hence, the original $v$ is not optimal. Similarly, the case where $v_{i} \geq\left\lceil c_{2}\right\rceil+1$ for some $i$ is ruled out.

Thus it follows that the optimal $v$ has $v_{i}=\left\lfloor c_{2}\right\rfloor$ for $p=\left(n_{1}-1\right)\left(\left\lceil c_{2}\right\rceil-c_{2}\right)$ values of $i$, and $v_{i}=\left\lceil c_{2}\right\rceil$ for the remaining $i$. The value for $d$ now easily follows from Lemma 1 .

For a graphical representation of the maximin distance as function of $n_{1}$ and $n_{2}$, see Fig. 1. Using the above results, a nested maximin design can easily be constructed: 


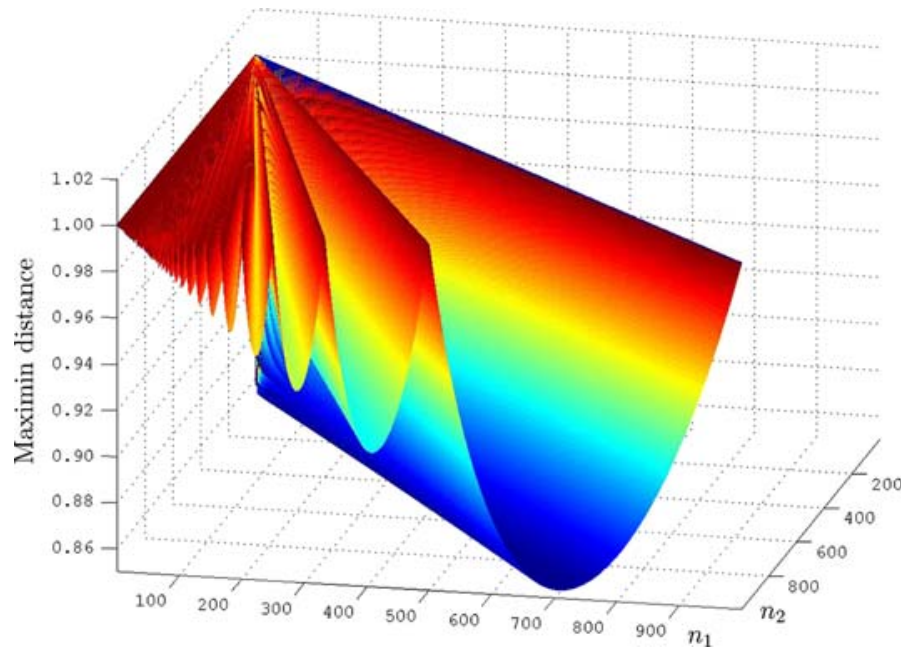

Fig. 1 Maximin distance as function of $n_{1}$ and $n_{2}$

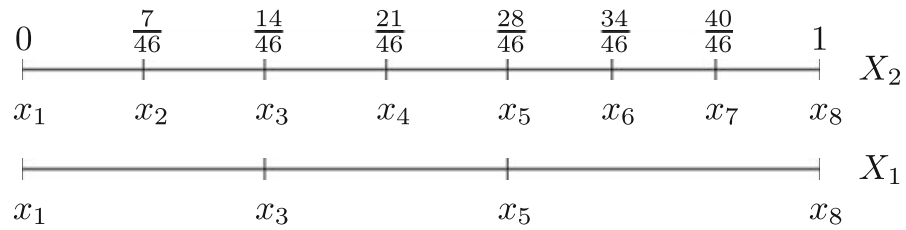

Fig. 2 A nested maximin design for $n_{1}=4$ and $n_{2}=8$, with $d=\frac{21}{23} \simeq 0.9130$

Construction 1 Let $2 \leq n_{1} \leq n_{2}$. A nested maximin design, with maximin distance $d$ as in (3), is given by

$$
\begin{aligned}
x_{j+1}= & \begin{cases}\frac{d}{n_{1}-1} \frac{j}{\left\lfloor c_{2}\right\rfloor} & j=0, \ldots, p\left\lfloor c_{2}\right\rfloor ; \\
\frac{d}{n_{1}-1} p+\frac{d}{n_{2}-1}\left(j-p\left\lfloor c_{2}\right\rfloor\right) & j=p\left\lfloor c_{2}\right\rfloor+1, \ldots, n_{2}-1 ;\end{cases} \\
I_{1}= & \left\{1+j\left\lfloor c_{2}\right\rfloor \mid j=0, \ldots, p\right\} \bigcup \\
& \left\{1+p\left\lfloor c_{2}\right\rfloor+(j-p)\left\lceil c_{2}\right\rceil \mid j=p+1, \ldots, n_{1}-1\right\} .
\end{aligned}
$$

As an example, we construct a nested maximin design for $n_{1}=4$ and $n_{2}=8$. From (3) we get that the maximin distance equals $d=\frac{21}{23} \simeq 0.9130$. Substituting $d$ and $p=2$ in (4) results in the points $x_{1}=0, x_{2}=\frac{7}{46}, x_{3}=\frac{14}{46}, x_{4}=\frac{21}{46}, x_{5}=\frac{28}{46}, x_{6}=\frac{34}{46}, x_{7}=\frac{40}{46}$, and $x_{8}=1$, and yields the set $I_{1}=\{1,3,5,8\}$, implying that $X_{1}=\left\{x_{1}, x_{3}, x_{5}, x_{8}\right\}$. See Fig. 2 for a graphical representation of this nested maximin design.

Besides computing the maximin distance for a given $n_{1}$ and $n_{2}$, (3) can also be used to prove a general lower bound on the maximin distance.

Proposition 2 Let $2 \leq n_{1} \leq n_{2}$. Then $1 \geq d>(4-2 \sqrt{2})^{-1} \simeq 0.853553$.

Proof Consider the function $z:[1, \infty) \rightarrow \mathbb{R}$ given by

$$
z\left(c_{2}\right)=1+\left\lfloor c_{2}\right\rfloor+\left\lceil c_{2}\right\rceil-c_{2}-\left\lfloor c_{2}\right\rfloor\left\lceil c_{2}\right\rceil \frac{1}{c_{2}}=1+\frac{\left(c_{2}-\left\lfloor c_{2}\right\rfloor\right)\left(\left\lceil c_{2}\right\rceil-c_{2}\right)}{c_{2}} \geq 1 .
$$


If $c_{2} \in \mathbb{N}$ then $z\left(c_{2}\right)=1$, i.e., $z$ is minimal and hence $d=z\left(c_{2}\right)^{-1} \leq 1$, else

$$
z\left(c_{2}+1\right)=1+\frac{\left(c_{2}-\left\lfloor c_{2}\right\rfloor\right)\left(\left\lceil c_{2}\right\rceil-c_{2}\right)}{c_{2}+1}<z\left(c_{2}\right) ; \quad c_{2} \notin \mathbb{N} .
$$

Therefore, in this case $z$ is maximal for some $c_{2} \in(1,2)$. Restrict $z$ to $(1,2)$ :

$$
z\left(c_{2}\right)=1+1+2-c_{2}-\frac{2}{c_{2}}=4-c_{2}-\frac{2}{c_{2}},
$$

which is maximal for $c_{2}=\sqrt{2}$. For $c_{2} \in \mathbb{Q}, c_{2} \geq 1$ :

$z\left(c_{2}\right)<z(\sqrt{2})=4-2 \sqrt{2}, \quad$ and then $\quad d>\frac{1}{z(\sqrt{2})}=\frac{1}{4-2 \sqrt{2}}=\frac{1}{2}+\frac{1}{4} \sqrt{2} \simeq 0.853553$.

Note that the obtained lower bound is tight since we can take $c_{2}$ arbitrarily close to $\sqrt{2}$. The interpretation of this lower bound is that for all values of $n_{1}$ and $n_{2}$, by nesting the sets $X_{1}$ and $X_{2}$ we will never lose more than $14.64 \%$, with respect to the "restriction free" maximin distance. In practice this implies that a linking parameter can be included in the maximin designs, or the designs can be used as training and test sets, at a cost of using designs that are at most $14.64 \%$ worse with respect to space-fillingness.

In case of sequential evaluations the interpretation is somewhat different. A standard way to perform (two-stage) sequential evaluations is to first choose $n_{1}$ points, equidistantly distributed over the interval $[0,1]$. After the evaluations, if needed, $n_{2}-n_{1}$ extra points are taken, resulting in $d^{\prime}=\frac{c_{2}}{\left\lceil c_{2}\right\rceil}$; see Sect. 2.2. Clearly, $d \geq d^{\prime}$ and $d^{\prime}=\frac{c_{2}}{\left\lceil c_{2}\right\rceil} \geq \frac{c_{2}}{c_{2}+1}>\frac{1}{2}$, for $c_{2}>1$. If one evaluation stage turns out to be sufficient, using the points in (1) will result in a design that is at most $14.64 \%$ worse than the (standard) equidistant design (since we lose $1-d$ ). However, if a second evaluation stage is needed then our approach results in a better space-filling design ( since we win $d-d^{\prime}$ ). Figure 3 shows the net gain of our approach, i.e., $(d-1)+\left(d-d^{\prime}\right)$, as function of $c_{2}$. For $n_{2} \leq 100$ the net gain takes values in the interval $[-0.07,0.48]$.

\subsection{Dominance}

In the last section we appraised the sets $X_{1}$ and $X_{2}$ to be equally important. What if one set is more important than the other? Or, given a fixed value for $d_{1}$, what is the corresponding maximal value of $d_{2}$ ? To examine this, we first introduce the notion of dominance. We will call a combination $\left(d_{1}, d_{2}\right)$ dominant if it is not possible to improve one of the coordinates, without deteriorating the other coordinate. Knowing the dominant combinations is very useful in practice. It enables us to determine the trade-off between $d_{1}$ and $d_{2}$, i.e., it helps us finding a combination that best satisfies our requirements, like " $X_{2}$ is more important than $X_{1}$ ". Note that the maximin combination $(d, d)$, with $d$ as in (3), is dominant. The combinations $\left(1, \frac{c_{2}}{\left\lceil c_{2}\right\rceil}\right)$ and $\left(\frac{\left\lfloor c_{2}\right\rfloor}{c_{2}}, 1\right)$ are also dominant, which can be argued as follows:

- Fixing $d_{1}=1$, the points of $X_{1}$ must be equidistantly distributed, i.e., $X_{1}=\left\{0, \frac{1}{n_{1}-1}\right.$, $\left.\frac{2}{n_{1}-1}, \ldots, 1\right\}$. Due to the restriction $I_{1} \subseteq I_{2}$ we need to find settings for the $n_{2}-n_{1}$ extra points in $X_{2}$, such that $d_{2}$ is maximal. This is accomplished by choosing these $n_{2}-n_{1}$ points as equally as possible spread over the $n_{1}-1$ intervals formed by the points in $X_{1}$, which corresponds to $v$ taking only the values $\left\lfloor c_{2}\right\rfloor$ and $\left\lceil c_{2}\right\rceil$, as before. Hence, after scaling, this gives a minimal distance of 


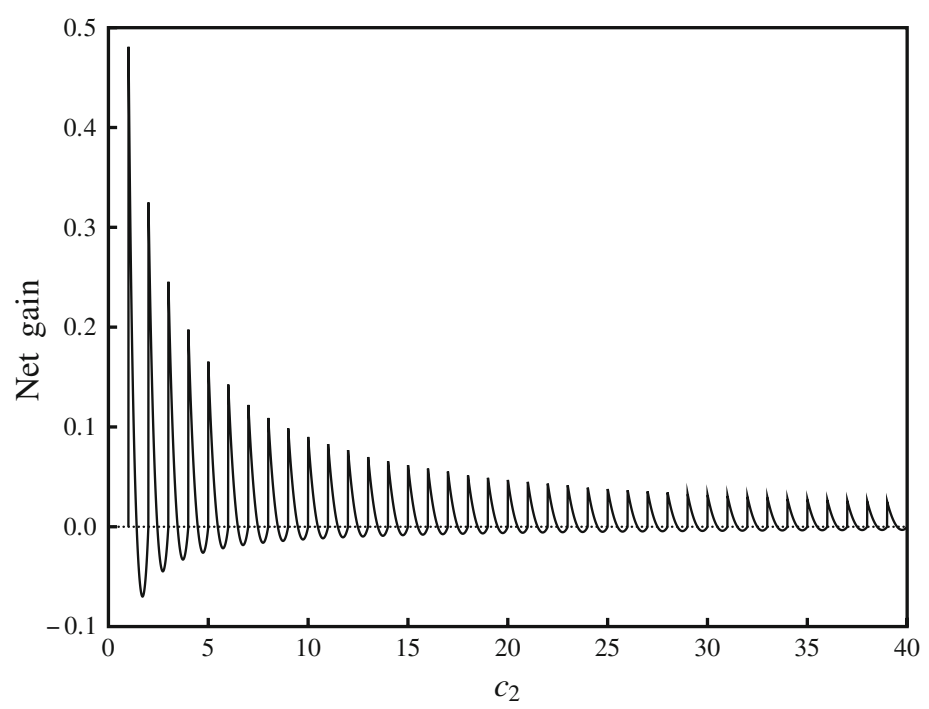

Fig. 3 Net gain of our approach as function of $c_{2}$

$$
d=d_{2}=\left(n_{2}-1\right)\left(\frac{1}{n_{1}-1} \cdot \frac{1}{\left\lceil c_{2}\right\rceil}\right)=\frac{c_{2}}{\left\lceil c_{2}\right\rceil} .
$$

- Fixing $d_{2}=1$, the points of $X_{2}$ must be equidistantly distributed, i.e., $X_{2}=\left\{0, \frac{1}{n_{2}-1}\right.$, $\left.\frac{2}{n_{2}-1}, \ldots, 1\right\}$. To maximize $d_{1}$, the $n_{2}-1$ intervals must as equally as possible be spread over the $n_{1}-1$ intervals that are to be formed by the points in $X_{1}$. Every interval of $X_{1}$ will then contain either $\left\lfloor c_{2}\right\rfloor$ or $\left\lceil c_{2}\right\rceil$ intervals of length $\frac{1}{n_{2}-1}$, and the minimal distance, after scaling, will be given by

$$
d=d_{1}=\left(n_{1}-1\right)\left(\frac{1}{n_{2}-1}\left\lfloor c_{2}\right\rfloor\right)=\frac{\left\lfloor c_{2}\right\rfloor}{c_{2}} .
$$

Since $\left(1, \frac{c_{2}}{\left\lceil c_{2}\right\rceil}\right)$ and $\left(\frac{\left\lfloor c_{2}\right\rfloor}{c_{2}}, 1\right)$ bound the values of $d_{1}$ and $d_{2}$ we will call them extreme dominant combinations. Moreover, note that these bounds imply that $d \geq \max \left\{\frac{c_{2}}{\left\lceil c_{2}\right\rceil}, \frac{\left\lfloor c_{2}\right\rfloor}{c_{2}}\right\}$. For a given $n_{1}$ and $n_{2}$ all dominant combinations can be characterized by the following linear function.

Proposition 3 Let $2 \leq n_{1} \leq n_{2}$. All dominant combinations $\left(d_{1}, d_{2}\right)$ are characterized by the linear function $f:\left[\frac{\left\lfloor c_{2}\right\rfloor}{c_{2}}, 1\right] \rightarrow\left[\frac{c_{2}}{\left\lceil c_{2}\right\rceil}, 1\right]$, where

$$
d_{2}=f\left(d_{1}\right)=\left(\left(c_{2}-\left\lceil c_{2}\right\rceil\right) d_{1}+1\right) \frac{c_{2}}{\left\lceil c_{2}\right\rceil\left(c_{2}-\left\lfloor c_{2}\right\rfloor\right)} .
$$

Proof Like in Lemma 1, we have for a given $I_{1}$, and corresponding $a, v$, that

$$
1 \geq \sum_{i=1}^{n_{1}-1} \max \left\{\frac{v_{i}}{n_{2}-1} d_{2}, \frac{1}{n_{1}-1} d_{1}\right\} .
$$

Hence, for a given $a, v$, and $d_{1} \leq 1$, it is optimal to choose $d_{2}$ as large as possible, such that equality is attained in (6). 
We claim that for any $d_{1}$, with $\frac{\left\lfloor c_{2}\right\rfloor}{c_{2}} \leq d_{1} \leq 1$, a maximal $d_{2}$ is obtained by letting $v$ take only the values $\left\lfloor c_{2}\right\rfloor$ and $\left\lceil c_{2}\right\rceil$, just like in Proposition 1 . Note that this needs no further proof for $d_{1}=\frac{\left\lfloor c_{2}\right\rfloor}{c_{2}}$ and $d_{1}=1$, therefore we may assume that $\frac{\left\lfloor c_{2}\right\rfloor}{c_{2}}<d_{1}<1$, and, hence, that $c_{2}$ is not an integer.

To prove the claim, fix $d_{1}$, and suppose that there is a $v$ giving an optimal $d_{2}$ with $v_{i} \geq\left\lceil c_{2}\right\rceil+1$ for some $i$. Let $j$ be such that $v_{j} \leq\left\lfloor c_{2}\right\rfloor$ (such a $j$ exists). Since $d_{2}$ is optimal we may assume that $d_{2} \geq \frac{c_{2}}{\left\lceil c_{2}\right\rceil}$. Now let $v^{\prime}$ be obtained from $v$ by subtracting 1 from $v_{i}$, and adding 1 to $v_{j}$. Since $d_{2}$ is optimal, the $d_{2}^{\prime}$ corresponding to $v^{\prime}$ is at most $d_{2}$.

From the equalities in (6) for the pairs $\left(v, d_{2}\right)$ and $\left(v^{\prime}, d_{2}^{\prime}\right)$, and the inequality $d_{2}^{\prime} \leq d_{2}$, we obtain that

$$
\begin{aligned}
& \max \left\{\frac{v_{i}}{n_{2}-1} d_{2}, \frac{1}{n_{1}-1} d_{1}\right\}+\max \left\{\frac{v_{j}}{n_{2}-1} d_{2}, \frac{1}{n_{1}-1} d_{1}\right\} \\
\leq & \max \left\{\frac{v_{i}-1}{n_{2}-1} d_{2}, \frac{1}{n_{1}-1} d_{1}\right\}+\max \left\{\frac{v_{j}+1}{n_{2}-1} d_{2}, \frac{1}{n_{1}-1} d_{1}\right\} .
\end{aligned}
$$

Because of the inequalities $v_{i} \geq\left\lceil c_{2}\right\rceil+1, v_{j} \leq\left\lfloor c_{2}\right\rfloor, 1 \geq d_{2} \geq \frac{c_{2}}{\left\lceil c_{2}\right\rceil}$, and $\frac{\left\lfloor c_{2}\right\rfloor}{c_{2}}<d_{1}<1$, this reduces to

$$
\frac{v_{i}}{n_{2}-1} d_{2}+\frac{1}{n_{1}-1} d_{1} \leq \frac{v_{i}-1}{n_{2}-1} d_{2}+\max \left\{\frac{v_{j}+1}{n_{2}-1} d_{2}, \frac{1}{n_{1}-1} d_{1}\right\} .
$$

Now this implies that $\frac{v_{j}+1}{n_{2}-1} d_{2} \geq \frac{1}{n_{1}-1} d_{1}$, and, hence, the inequality further reduces to $\frac{1}{n_{1}-1} d_{1} \leq \frac{v_{j}}{n_{2}-1} d_{2}$. Using that $\frac{\left\lfloor c_{2}\right\rfloor}{c_{2}}<d_{1}$ and $d_{2} \leq 1$, this implies that $v_{j}>\left\lfloor c_{2}\right\rfloor$, which is a contradiction, hence, the considered $v$ does not give an optimal $d_{2}$. Similarly, it can be shown that the case where $v_{i}<\left\lfloor c_{2}\right\rfloor$ for some $i$ is not optimal.

Thus, for any $d_{1}$ it is optimal to take $a$ such that $v_{i}=\left\lfloor c_{2}\right\rfloor$ for $p=\left(n_{1}-1\right)\left(\left\lceil c_{2}\right\rceil-c_{2}\right)$ values of $i$, and $v_{i}=\left\lceil c_{2}\right\rceil$ for the remaining $i$. The value for $d_{2}$ as a function of $d_{1}$ now easily follows from equality in (6).

We remark that for fixed $a$ and $v$, the relation between $d_{1}$ and $d_{2}$ can be found by considering equality in (6). This relation will be a piece-wise linear function. Further, note that for $c_{2} \in \mathbb{N}$ the graph of (5) results in the single point $(1,1)$, and that setting $d_{1}=d_{2}$ in (5) yields the maximin distance $d$, with $d$ as in (3). See Fig. 4 for a graphical example of the linear function $f$. This figure shows the set of dominant combinations for $n_{1}=4$ and $n_{2}=8$, including the two extreme dominant combinations $\left(1, \frac{c_{2}}{\left\lceil c_{2}\right\rceil}\right)=(1,0.7778)$ and $\left(\frac{\left\lfloor c_{2}\right\rfloor}{c_{2}}, 1\right)=(0.8571,1)$. Moreover, the line $d_{1}=d_{2}$ intersects the dominant set exactly in the maximin combination $(d, d)=(0.9130,0.9130)$.

\section{Three nested sets}

We now discuss the case of three nested sets, i.e., $m=3$. Section 3.1 starts with the general problem formulation. Since we are not able to come up with an explicit formula for the maximin distance we use mixed integer linear programming to solve the problem for several $n_{1}, n_{2}, n_{3}$. Fortunately, a lower bound on the maximin distance can still be proven. Section 3.2 discusses dominant combinations and in Sect. 3.3 a heuristic that yields extremely good nested designs is developed. 


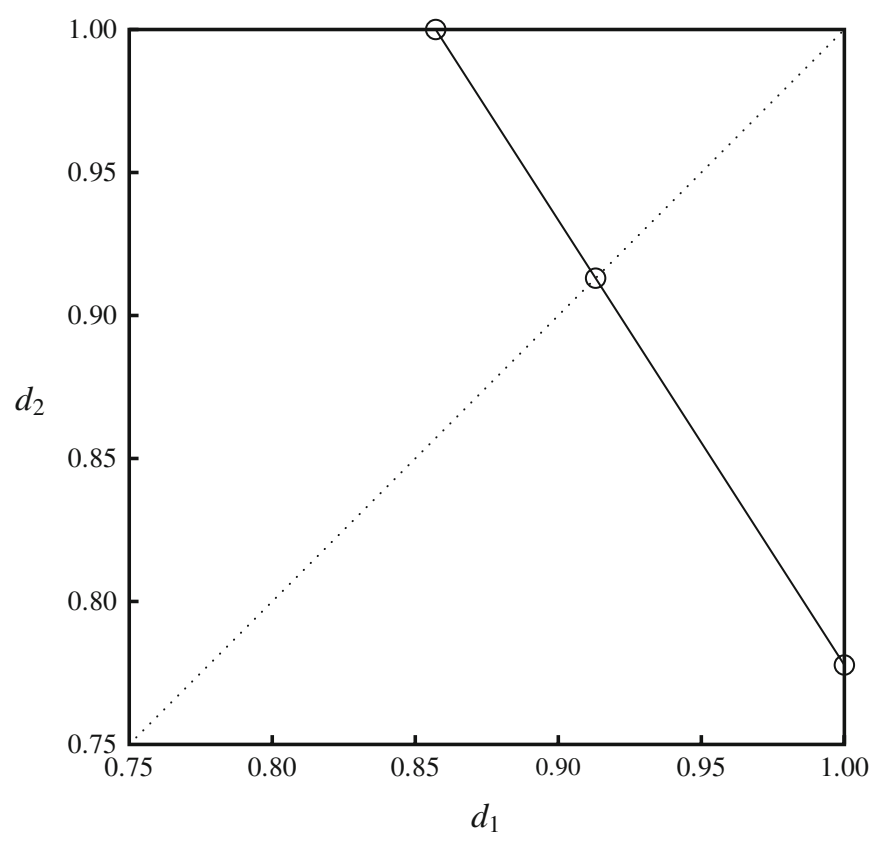

Fig. 4 All dominant combinations $\left(d_{1}, d_{2}\right)$ for $n_{1}=4$ and $n_{2}=8$, and the line $d_{1}=d_{2}$

\subsection{Maximin distance}

The general problem for three nested sets can be formalized as the following mathematical program:

$$
\begin{array}{lll}
\max & \min _{\substack{j, k \in I_{i} \\
i=1,2,3 ; j \neq k}}\left(n_{i}-1\right)\left|x_{j}-x_{k}\right| & \\
& & \\
\text { s.t. } & I_{1} \subseteq I_{2} \subseteq I_{3}=\left\{1, \ldots, n_{3}\right\} & \\
& \left|I_{i}\right|=n_{i}, & i=1,2 \\
& 0 \leq x_{j} \leq 1, & j \in I_{3} .
\end{array}
$$

As in Sect. 2.1 we may choose without loss of generality $x_{1}=0, x_{n_{3}}=1, x_{i}<x_{i+1}, 1 \in I_{1}$, $n_{3} \in I_{1}, 1 \in I_{2}$, and $n_{3} \in I_{2}$. For a given $I_{2}$, containing the indices, say, $1=b_{1}<b_{2}<$ $\cdots<b_{n_{2}}=n_{3}$ we introduce the sequence $w=\left(w_{1}, \ldots, w_{n_{2}-1}\right)$ given by $w_{j}=b_{j+1}-b_{j}$. Given an $I_{1}$ contained in this $I_{2}$ we let $1=a_{1}<a_{2}<\cdots<a_{n_{1}}=n_{2}$ be such that $b_{a_{i}} \in I_{1}$ for $i=1, \ldots, n_{1}$. Notice that in this case $\left\{a_{i} \mid i=1, \ldots, n_{1}\right\} \neq I_{1}$. Analogously to the definition introduced in Sect. 2.1, we let $v_{i}=a_{i+1}-a_{i}$. Thus $v_{i}-1$ gives the number of additional points of $X_{2}$ between the $i$-th and $(i+1)$-st point of $X_{1}$, while $w_{j}-1$ gives the number of additional points of $X_{3}$ between the $j$-th and $(j+1)$-st point of $X_{2}$. Now the analogue of Lemma 1 is the following.

Lemma 2 For fixed $I_{1}, I_{2}$, and corresponding $a, b, v, w$, the optimal value $\delta_{a, w}$ equals

$$
\left(\sum_{i=1}^{n_{1}-1} \max \left\{\sum_{j=a_{i}}^{a_{i+1}-1} \max \left\{\frac{w_{j}}{n_{3}-1}, \frac{1}{n_{2}-1}\right\}, \frac{1}{n_{1}-1}\right\}\right)^{-1} .
$$




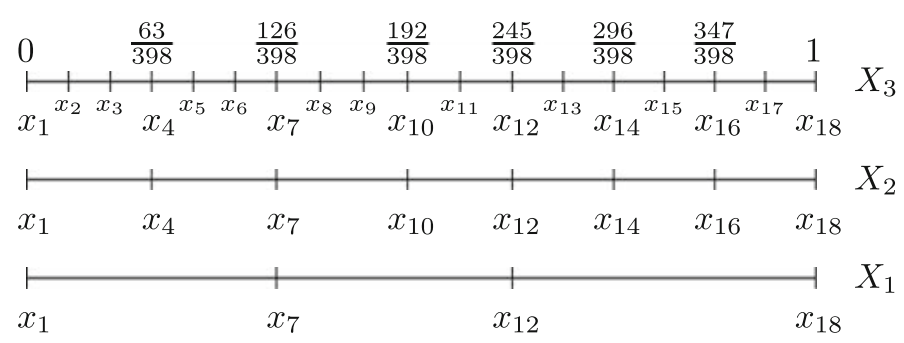

Fig. 5 A nested maximin design for $n_{1}=4, n_{2}=8$, and $n_{3}=18$, with $d=\frac{357}{398} \simeq 0.8970$

We would now have to maximize $\delta_{a, w}$ over all appropriate sequences $a$ and $w$. Unfortunately, we are not able to come up with an explicit formula for the maximin distance, as we did for two nested sets in Sect. 2.1. However, we can rewrite (7) as a mixed integer linear program:

$$
\begin{array}{lll}
\max & d & \\
\text { s.t. } & d \leq\left(n_{3}-1\right)\left(x_{j+1}-x_{j}\right), & j \in I_{3} \backslash\left\{n_{3}\right\} \\
& d \leq\left(n_{i}-1\right)\left(x_{k}-x_{j}\right)+2-z_{i k}-z_{i j}, & i=1,2 ; j, k \in I_{3} ; j<k \\
& \sum_{j=1}^{n_{3}} z_{i j}=n_{i}, & i=1,2 \\
& z_{1 j} \leq z_{2 j}, & j \in I_{3} \\
& 0 \leq x_{j} \leq 1, & j \in I_{3} \\
& z_{i j} \in\{0,1\}, & i=1,2 ; j \in I_{3} .
\end{array}
$$

Here, $z_{i j}=1$ if $j \in I_{i}$, and $z_{i j}=0$ otherwise. The constraints $\sum_{j=1}^{n_{3}} z_{i j}=n_{i}$ and $z_{1 j} \leq z_{2 j}$ insure that $\left|I_{i}\right|=n_{i}$ and $I_{1} \subseteq I_{2}$, respectively. Using (8) and the XA Mixed Integer Solver we found results up to $n_{3}=25$, with computation times varying from 1 second to almost $2.5 \mathrm{~h}$ for some instances, on a PC with an $800-\mathrm{MHz}$ Pentium III processor.

As an example of a nested maximin design, take $n_{1}=4, n_{2}=8$, and $n_{3}=18$. Solving (8) for this instance yields the sets $I_{1}=\{1,7,12,18\}$ and $I_{2}=\{1,4,7,10,12,14,16,18\}$, implying that $X_{1}=\left\{x_{1}, x_{7}, x_{12}, x_{18}\right\}$ and $X_{2}=\left\{x_{1}, x_{4}, x_{7}, x_{10}, x_{12}, x_{14}, x_{16}, x_{18}\right\}$, which gives $d=\frac{357}{398} \simeq 0.8970$. See Fig. 5 for a graphical representation of the design.

Although we do not have an explicit formula for the maximin distance, we can prove a general lower bound on this distance. To accomplish this, let $d\left(n_{1}, n_{2}, n_{3}\right)$ be the optimal value for $d$ as function of $n_{1}, n_{2}, n_{3}$, and consider the following lemma.

Lemma 3 Let $2 \leq n_{1} \leq n_{2} \leq n_{3}$. Then $d\left(n_{1}, n_{2}, n_{3}\right) \leq d\left(n_{1}, n_{2}, n_{3}+n_{2}-1\right)$.

Proof Consider any $a$ and $w$ for the problem of $\left(n_{1}, n_{2}, n_{3}\right)$. For the problem of $\left(n_{1}, n_{2}, n_{3}+\right.$ $n_{2}-1$ ) we consider the same $a$, and $w^{\prime}$ which is given by $w_{j}^{\prime}=w_{j}+1$ for all $j$. Since

$$
\max \left\{\frac{w_{j}+1}{n_{3}+n_{2}-1-1}, \frac{1}{n_{2}-1}\right\} \leq \max \left\{\frac{w_{j}}{n_{3}-1}, \frac{1}{n_{2}-1}\right\},
$$

which is easy to show, this implies that $\delta_{a, w^{\prime}}\left(n_{1}, n_{2}, n_{3}+n_{2}-1\right) \geq \delta_{a, w}\left(n_{1}, n_{2}, n_{3}\right)$, and the result follows.

Proposition 4 Let $2 \leq n_{1} \leq n_{2} \leq n_{3}$. Then $1 \geq d\left(n_{1}, n_{2}, n_{3}\right)>(6-3 \sqrt[3]{4})^{-1} \simeq 0.807887$.

Proof Let (again) $c_{2}=\frac{n_{2}-1}{n_{1}-1}$ and $c_{3}=\frac{n_{3}-1}{n_{2}-1}$. First, note that $d\left(n_{1}, n_{2}, n_{3}\right)=1$ if and only if $c_{2}, c_{3} \in \mathbb{N}$. Because of Lemma 3 , we may assume without loss of generality that 
$c_{3}<2$. To prove the stated inequality, we shall give an $a$ and $w$ such that $\delta_{a, w}\left(n_{1}, n_{2}, n_{3}\right)>$ $(6-3 \sqrt[3]{4})^{-1}$.

Let $a$ be such that the corresponding $v$ takes the value $v_{i}=\left\lfloor c_{2}\right\rfloor$ for $i=1, \ldots, p$, with $p=\left(n_{1}-1\right)\left(\left\lceil c_{2}\right\rceil-c_{2}\right)$, and $v_{i}=\left\lceil c_{2}\right\rceil$ for the remaining $i$, i.e., it is the optimal $a$ for two nested sets. Since $c_{3}<2$, it is possible to take $w$ such that $w_{j}$ is one or two for all $j$, and we shall do so. To further describe $w$, we distinguish between two cases.

If $n_{3}-n_{2} \geq\left\lfloor c_{2}\right\rfloor\left(n_{1}-1\right)\left(\left\lceil c_{2}\right\rceil-c_{2}\right)$, then we let $w$ be such that $w_{j}=2$ for $j=1, \ldots, n_{3}-n_{2}$, and $w_{j}=1$ for the remaining $j$. Since $\max \left\{\frac{2}{n_{3}-1}, \frac{1}{n_{2}-1}\right\}=\frac{2}{n_{3}-1}$, we have that

$$
\begin{aligned}
\delta_{a, w}^{-1}= & \left(n_{1}-1\right)\left(\left\lceil c_{2}\right\rceil-c_{2}\right) \max \left\{\frac{2\left\lfloor c_{2}\right\rfloor}{n_{3}-1}, \frac{1}{n_{1}-1}\right\} \\
& +\left(n_{3}-n_{2}-\left\lfloor c_{2}\right\rfloor\left(n_{1}-1\right)\left(\left\lceil c_{2}\right\rceil-c_{2}\right)\right) \frac{2}{n_{3}-1}+\left(n_{2}-1-\left(n_{3}-n_{2}\right)\right) \frac{1}{n_{2}-1} \\
= & \left(\left\lceil c_{2}\right\rceil-c_{2}\right) \max \left\{0,1-\frac{2\left\lfloor c_{2}\right\rfloor}{c_{2} c_{3}}\right\}+4-c_{3}-\frac{2}{c_{3}} .
\end{aligned}
$$

Thus, if $2\left\lfloor c_{2}\right\rfloor<c_{2} c_{3}$, then $\delta_{a, w}^{-1}=\left(\left\lceil c_{2}\right\rceil-c_{2}\right)\left(1-\frac{2\left\lfloor c_{2}\right\rfloor}{c_{2} c_{3}}\right)+4-c_{3}-\frac{2}{c_{3}}$. Call this expression $f\left(c_{2}\right)$, then it is easy to see that $f\left(c_{2}+1\right)<f\left(c_{2}\right)$, hence, we may restrict our attention to the case where $1<c_{2}<2$. From the above we now obtain that $\delta_{a, w}^{-1}=6-c_{2}-c_{3}-\frac{4}{c_{2} c_{3}}$. This expression is at most $6-3 \sqrt[3]{4}$, a value that is attained only if $c_{2}=c_{3}=\sqrt[3]{4}$. The case $2\left\lfloor c_{2}\right\rfloor \geq c_{2} c_{3}$ is straightforward (then $\delta_{a, w}^{-1} \leq 4-2 \sqrt{2}$ ), so for the case $n_{3}-n_{2} \geq$ $\left\lfloor c_{2}\right\rfloor\left(n_{1}-1\right)\left(\left\lceil c_{2}\right\rceil-c_{2}\right)$ we have proven the lower bound on $d$.

If $n_{3}-n_{2}<\left\lfloor c_{2}\right\rfloor\left(n_{1}-1\right)\left(\left\lceil c_{2}\right\rceil-c_{2}\right)$, then we may assume that $c_{2}$ is not an integer. Let $p=\left(n_{1}-1\right)\left(\left\lceil c_{2}\right\rceil-c_{2}\right)$, and introduce $t=\frac{n_{3}-n_{2}}{p}=\frac{c_{2}\left(c_{3}-1\right)}{\left\lceil c_{2}\right\rceil-c_{2}}$. It follows that $\lceil t\rceil \leq\left\lfloor c_{2}\right\rfloor$. We now take $w$ as follows: for $m(t-\lfloor t\rfloor)$ values of $i=1, \ldots, m$ we have $\lceil t\rceil$ values of $j$, $a_{i} \leq j<a_{i+1}$ for which $w_{j}=2$, and the remaining $\left\lfloor c_{2}\right\rfloor-\lceil t\rceil$ of such $j$-s have $w_{j}=1$; for the other values of $i=1, \ldots, m$ we have $\lfloor t\rfloor$ values of $j, a_{i} \leq j<a_{i+1}$ for which $w_{j}=2$, and the remaining $\left\lfloor c_{2}\right\rfloor-\lfloor t\rfloor$ of such $j$-s have $w_{j}=1$; and for all $j \geq a_{m+1}$ we have $w_{j}=1$. From Lemma 2, we now find that

$$
\begin{aligned}
\delta_{a, w}^{-1}= & m(t-\lfloor t\rfloor) \max \left\{\lceil t\rceil \frac{2}{n_{3}-1}+\left(\left\lfloor c_{2}\right\rfloor-\lceil t\rceil\right) \frac{1}{n_{2}-1}, \frac{1}{n_{1}-1}\right\} \\
& +m(1-t+\lfloor t\rfloor) \max \left\{\lfloor t\rfloor \frac{2}{n_{3}-1}+\left(\left\lfloor c_{2}\right\rfloor-\lfloor t\rfloor\right) \frac{1}{n_{2}-1}, \frac{1}{n_{1}-1}\right\} \\
& +\left(n_{1}-1\right)\left(c_{2}-\left\lfloor c_{2}\right\rfloor\right)\left\lceil c_{2}\right\rceil \frac{1}{n_{2}-1} \\
= & \frac{\left\lceil c_{2}\right\rceil-c_{2}}{c_{2}}(t-\lfloor t\rfloor) \max \left\{\lceil t\rceil\left(\frac{2}{c_{3}}-1\right), c_{2}-\left\lfloor c_{2}\right\rfloor\right\} \\
& +\frac{\left\lceil c_{2}\right\rceil-c_{2}}{c_{2}}(1-t+\lfloor t\rfloor) \max \left\{\lfloor t\rfloor\left(\frac{2}{c_{3}}-1\right), c_{2}-\left\lfloor c_{2}\right\rfloor\right\}+1 .
\end{aligned}
$$

We now assume that

$$
\lfloor t\rfloor\left(\frac{2}{c_{3}}-1\right)<c_{2}-\left\lfloor c_{2}\right\rfloor<\lceil t\rceil\left(\frac{2}{c_{3}}-1\right) .
$$


In the other cases it is straightforward to show that $\delta_{a, w}^{-1} \leq 4-2 \sqrt{2}$. Then

$$
\delta_{a, w}^{-1}=\frac{\left\lceil c_{2}\right\rceil-c_{2}}{c_{2}}(t-\lfloor t\rfloor)\lceil t\rceil\left(\frac{2}{c_{3}}-1\right)+\frac{\left\lceil c_{2}\right\rceil-c_{2}}{c_{2}}(1-t+\lfloor t\rfloor)\left(c_{2}-\left\lfloor c_{2}\right\rfloor\right)+1 .
$$

Using the left inequality in above assumption we find that

$$
\begin{aligned}
\delta_{a, w}^{-1} & <\frac{\left\lceil c_{2}\right\rceil-c_{2}}{c_{2}}(t-\lfloor t\rfloor)\left(\frac{2}{c_{3}}-1\right)+\frac{\left\lceil c_{2}\right\rceil-c_{2}}{c_{2}}\left(c_{2}-\left\lfloor c_{2}\right\rfloor\right)+1 \\
& \leq\left(c_{3}-1\right)\left(\frac{2}{c_{3}}-1\right)+\frac{\left\lceil c_{2}\right\rceil-c_{2}}{c_{2}}\left(c_{2}-\left\lfloor c_{2}\right\rfloor\right)+1 \\
& \leq 4-2 \sqrt{2}+\frac{\left\lceil c_{2}\right\rceil-c_{2}}{c_{2}}\left(c_{2}-\left\lfloor c_{2}\right\rfloor\right) .
\end{aligned}
$$

If $c_{2}>4$, then this upper bound suffices (its maximum is attained at $c_{2}=\sqrt{20}$ ), as one can easily check. For $c_{2}<4$, fix $k=\lceil t\rceil(\leq 3)$, and let $c_{2}>k$. Then (9) reduces to

$$
\delta_{a, w}^{-1}=1+3 k-k c_{3}-\frac{2 k}{c_{3}}-\left(c_{3}-1\right)\left(c_{2}-\left\lfloor c_{2}\right\rfloor\right)+k \frac{\left\lceil c_{2}\right\rceil-c_{2}}{c_{2}}\left(c_{2}-\left\lfloor c_{2}\right\rfloor-(k-1)\left(\frac{2}{c_{3}}-1\right)\right),
$$

the maximum of which is attained for some $c_{2}$ between $k$ and $k+1$, i.e., $\left\lfloor c_{2}\right\rfloor$ is minimal. For each $k=1,2,3$ (separately) it is now possible to obtain an appropriate upper bound on $\delta_{a, w}^{-1}$, under the assumptions that $k \leq c_{2} \leq k+1$ and $1 \leq c_{3} \leq 2$. For $k=1$, this upper bound is $6-3 \sqrt[3]{4}$, and it is attained when $c_{2}=c_{3}=\sqrt[3]{2}$.

Note that the obtained lower bound is tight since we can take $c_{2}$ and $c_{3}$ arbitrarily close to $\sqrt[3]{2}$, and in these cases the given $a$ and $w$ are optimal; see Proposition 6 . The interpretation of this lower bound is that for all values of $n_{1}, n_{2}, n_{3}$, by nesting the sets $X_{1}, X_{2}, X_{3}$ we will never lose more than $19.21 \%$, with respect to the "restriction free" maximin distance. In practice this implies that a linking parameter can be included in the maximin designs, at a cost of using designs that are at most $19.21 \%$ worse with respect to space-fillingness.

Applying our approach in case of (three-stage) sequential evaluations incurs a loss of $1-d$ when one stage suffices. If two stages are sufficient we obtain a net gain of $d-d^{\prime}$, where $d^{\prime}=\frac{c_{2}}{\left\lceil c_{2}\right\rceil}$ (see Sect. 2.1), and when all three stages are needed we gain $d-d^{\prime \prime}$, where $d^{\prime} \prime \leq d^{\prime} \leq d$ and $d^{\prime \prime}>\frac{1}{2}$ for $c_{3}>1$. Thus, the net gain of our approach equals $(d-1)+\left(d-d^{\prime}\right)+\left(d-d^{\prime \prime}\right)$ and it takes values in the interval $[-0.19,0.84]$ for $n_{3} \leq 25$.

\subsection{Dominance}

The notion of dominance was introduced in Sect. 2.2. Similar as before, we will call a combination $\left(d_{1}, d_{2}, d_{3}\right)$ dominant if it is not possible to improve one of the coordinates, without deteriorating another coordinate. Unlike with two nested sets, the maximin combination $(d, d, d)$ is not necessarily dominant, e.g., $d(4,8,17)=0.9130$, however, $(0.9130,0.9130$, 0.9275) is dominant. In Sect. 2.2, we showed that $\left(1, \frac{c_{2}}{\left\lceil c_{2}\right\rceil}\right)$ and $\left(\frac{\left\lfloor c_{2}\right\rfloor}{c_{2}}, 1\right)$ are extreme dominant combinations for two nested sets. Extending these ideas to three nested sets, i.e., fixing $d_{i}=1$ and maximizing $d_{j}, j \neq i$, leads to extreme dominant combinations. Note that the extreme dominant combinations are again lower bounds on the maximin distance $d=$ $d\left(n_{1}, n_{2}, n_{3}\right)$. An upper bound on $d$ is obtained by the simple observation that $d\left(n_{1}, n_{2}, n_{3}\right) \leq$ $\max \left\{d\left(n_{1}, n_{2}\right), d\left(n_{1}, n_{3}\right), d\left(n_{2}, n_{3}\right)\right\}$. Furthermore, it is easily shown that $d\left(n_{1}, n_{2}, n_{3}\right)=$ $d\left(n_{2}, n_{3}\right)$ if and only if $c_{2} \in \mathbb{N}$, and $d\left(n_{1}, n_{2}, n_{3}\right)=d\left(n_{1}, n_{2}\right)$ if and only if $c_{3} \in \mathbb{N}$. 


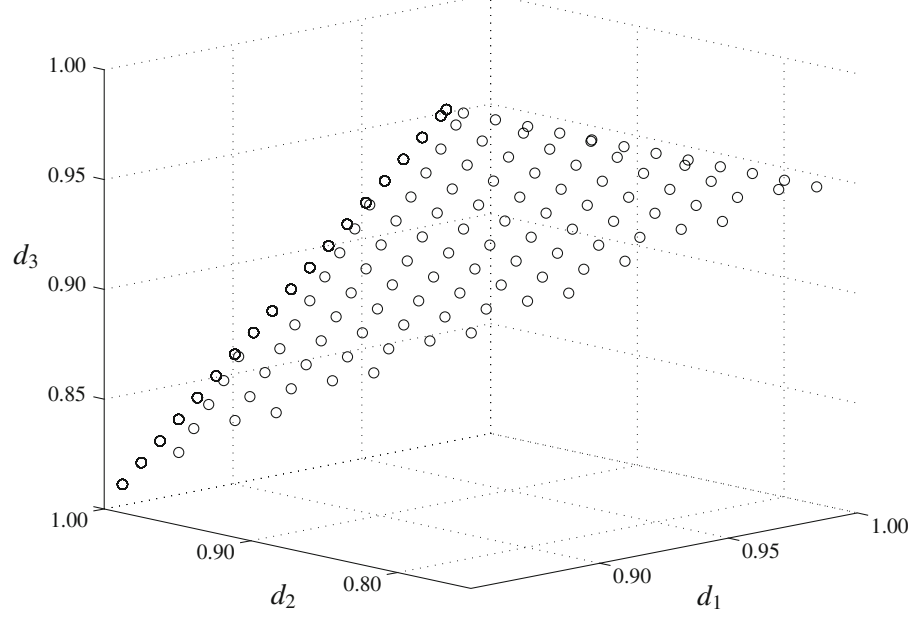

Fig. 6 Dominant combinations for $n_{1}=4, n_{2}=8$, and $n_{3}=18$

All this may lead to the believe that we can extend the idea of finding the maximin distance by means of extreme dominant combinations, like we did for two nested sets. As an example, from Fig. 6 it can be seen that the dominant combinations for $n_{1}=4, n_{2}=8$, and $n_{3}=18$, lie in a plane through the extreme dominant combinations $(1,0.7778,0.9444)$, $(0.8571,1,0.8095)$, and $(0.8824,0.8235,1)$. This plane intersects the line $d_{3}=d_{2}=d_{1}$ exactly in the maximin combination $(0.8970,0.8970,0.8970)$, strengthening the believe that this method also works for three nested sets. Unfortunately, the dominant combinations will not always fall in a plane through the extreme dominant points; see Fig. 7 for an example of this. Furthermore, this plane can not always be used to find the maximin combination. For example, take $n_{1}=6, n_{2}=8$, and $n_{3}=12$. Then the plane through the extreme dominant combinations $(1,0.7,0.7333),(0.7143,1,0.7857)$, and $(0.9091,0.6364,1)$, results in the unattainable combination $(0.8324,0.8324,0.8324)$, when intersected with the line $d_{3}=d_{2}=d_{1}$, thereby "missing" the correct maximin combination $(0.8262,0.8262$, $0.8262)$.

\subsection{Heuristic}

In the previous section we showed that, when dealing with three nested sets, the maximin distance cannot always be found by means of extreme dominant combinations. Note that even when this method would work, we still had to find a way to construct the corresponding nested maximin designs. Mixed integer linear programming can be used; however, it was found to be too slow in finding nested maximin designs for large values of $n_{1}, n_{2}, n_{3}$. We have also tried to solve (7) with the state-of-the-art global optimization software LGO, see Pintér (1995). Moreover, we have developed a heuristic that takes into account the special structure of the problem. It appeared that the solutions obtained with our tailored heuristic are much better than those obtained by the, more general, global optimization solver LGO.

Our heuristic is based on the observation that all nested maximin designs that were found by solving (8) contained the corresponding two nested sets assignments, as given in (4), as part of their solutions, e.g., compare Figs. 2 and 5. Therefore, for given $n_{1}, n_{2}, n_{3}\left(c_{2}, c_{3} \notin \mathbb{N}\right)$, 
we first use (1) to construct a nested maximin design on $n_{1}, n_{2}$. Every interval $\left[x_{l}, x_{l+1}\right]$, $l \in I_{2} \backslash\left\{n_{2}\right\}$, then will have a width of at least $\frac{d}{n_{2}-1}$, where $d=d\left(n_{1}, n_{2}\right)$ is as in (1). This implies that we can add up to $q$ points to each interval without decreasing $d$, as long as $q$ fulfills the inequality

$$
\frac{d(q+1)}{n_{3}-1} \leq \frac{d}{n_{2}-1}, \quad \text { or equivalently } q+1 \leq c_{3},
$$

which results in at most $q=\left\lfloor c_{3}\right\rfloor-1$ additional points per interval, or $\left(\left\lfloor c_{3}\right\rfloor-1\right)\left(n_{2}-1\right)$ in total. Hence, if $n_{3}-n_{2} \leq\left(\left\lfloor c_{3}\right\rfloor-1\right)\left(n_{2}-1\right)$, we are finished, since spreading the $n_{3}-n_{2}$ points equally over the $n_{2}-1$ intervals will yield a nested maximin design with distance $d\left(n_{1}, n_{2}, n_{3}\right)=d\left(n_{1}, n_{2}\right)$.

If $n_{3}-n_{2}>\left(\left\lfloor c_{3}\right\rfloor-1\right)\left(n_{2}-1\right)$, we add $q$ points to every interval and have $r=$ $\left(n_{3}-n_{2}\right)-\left(\left\lfloor c_{3}\right\rfloor-1\right)\left(n_{2}-1\right)<\left(n_{3}-n_{2}\right)-\left(c_{3}-2\right)\left(n_{2}-1\right)=n_{2}-1$ points remaining. These remaining $r$ points are then sequentially added to one of the $n_{2}-1$ intervals as follows. Consider the case where $s$ points are already assigned, $s \in\{0, \ldots, r-1\}$, and consider the index sets $I_{1}^{s} \subseteq I_{2}^{S} \subseteq I_{3}^{S}=\left\{1, \ldots, n_{3}^{\prime}\right\}$, which describe the current nested design on $n_{1}, n_{2}, n_{3}^{\prime}$, where $n_{3}^{\prime}=n_{2}+\left(\left\lfloor c_{3}\right\rfloor-1\right)\left(n_{2}-1\right)+s$. Then the corresponding maximal distance can readily be computed using Lemma 2 .

When assigning the $(s+1)$-st point we first compute for each of the $n_{2}-1$ intervals what the maximal distance will be if the point is assigned to that particular interval, again using Lemma 2. Naturally, the interval for which this distance is the largest is chosen and the corresponding $I_{1}^{s+1} \subseteq I_{2}^{s+1} \subseteq I_{3}^{s+1}$ describe the new nested design. In case of a tie we choose that interval for which

$$
\max \left\{\frac{\left(I_{2}^{s+1}\right)_{i+1}-\left(I_{2}^{s+1}\right)_{i}}{n_{3}-1}, \frac{1}{n_{2}-1}\right\}-\max \left\{\frac{\left(I_{2}^{s}\right)_{i+1}-\left(I_{2}^{s}\right)_{i}}{n_{3}-1}, \frac{1}{n_{2}-1}\right\}
$$

is the smallest, $i=1, \ldots, n_{2}-1$. Here, $\left(I_{2}^{s}\right)_{i}$ and $\left(I_{2}^{s+1}\right)_{i}$ are defined as the $i$-th smallest elements of the sets $I_{2}^{S}$ and $I_{2}^{s+1}$, respectively. This value can be seen as the relative cost of adding an extra point to a particular interval. Leaving out this second objective may result in bad nested designs.

For given index sets $I_{1}, I_{2}, I_{3}$ it takes $\mathcal{O}\left(n_{1} n_{2}\right)$ time to compute the maximal distance, using Lemma 2. There are $s \leq r<n_{2}$ extra points to be added and for each of these points $n_{2}-1$ index sets have to be considered, hence, we have to apply Lemma $2 \mathcal{O}\left(n_{2}{ }^{2}\right)$ times. Therefore, a nested design for $n_{1}, n_{2}, n_{3}$ is found in $\mathcal{O}\left(n_{1} n_{2}{ }^{3}\right)$ time. Note that the complexity does not depend on $n_{3}$. Moreover, it turns out that our heuristic yields an optimal nested design for all values of $n_{1}, n_{2}, n_{3}$ we solved so far, i.e., for $n_{3} \leq 25$. We conjecture that the heuristic will find a nested maximin design for all $n_{1}, n_{2}, n_{3}$.

\section{Four or more nested sets}

In this section, we discuss the case of $m \geq 4$ nested sets. This can be formalized as the following mathematical program: 


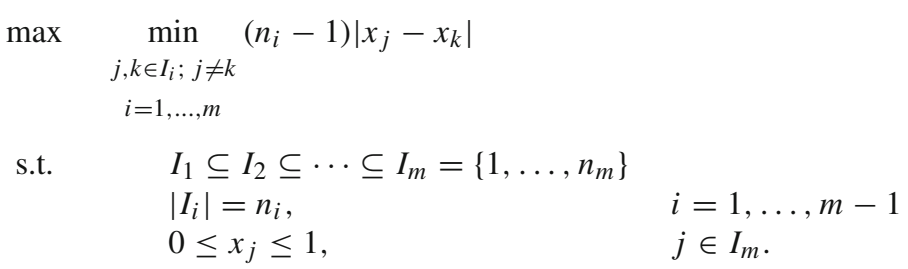

Furthermore, Lemmas 2 and 3 can easily be generalized to more nested sets. In particular,

Lemma 4 Let $2 \leq n_{1} \leq \cdots \leq n_{m}$. Then $d\left(n_{1}, \ldots, n_{m-1}, n_{m}\right) \leq d\left(n_{1}, \ldots, n_{m-1}, n_{m}+\right.$ $\left.n_{m-1}-1\right)$.

Now, we consider the case $n_{m}<2 n_{1}$. Let $c_{i}=\frac{n_{i}-1}{n_{i-1}-1}, i=2, \ldots, m$, and $d=$ $d\left(n_{1}, \ldots, n_{m}\right)$. For fixed $I_{1}$, let it contain the indices $1=a_{1}<a_{2}<\cdots<a_{n_{1}}=n_{m}$. Note that this $a$ is somewhat different from $a$ in the previous section, in the sense that it here gives the relation between $I_{1}$ and $I_{m}$, instead of between $I_{1}$ and $I_{2}$. As before, let the sequence $v=\left(v_{1}, \ldots, v_{n_{1}-1}\right)$ be given by $v_{i}=a_{i+1}-a_{i}$. Thus $v_{i}-1$ gives the number of additional points of $X_{m}$ between the $i$-th and $(i+1)$-st point of $X_{1}$.

Proposition 5 Let $m \geq 3$ and $2 \leq n_{1} \leq \cdots \leq n_{m}<2 n_{1}$. Then the maximal value for $d$ equals

$$
\frac{1}{2 m-\frac{2}{c_{2}}-\cdots-\frac{2}{c_{m}}-c_{2} c_{3} \cdots c_{m}} .
$$

Proof Consider an $I_{1}$ such that the corresponding $v$ takes only values 1 and 2, i.e., between two neighboring points from $X_{1}$ there is at most one point from $X_{m}$. Since $\max \left\{\frac{2}{n_{j}-1}, \frac{1}{n_{1}-1}\right\}$ $=\frac{2}{n_{j}-1}$, and since the number of $i$ such that $v_{i}=1$ equals $2 n_{1}-n_{m}-1$, it follows that

$$
\begin{aligned}
\delta_{v} & =\left(\left(2 n_{1}-n_{m}-1\right) \frac{1}{n_{1}-1}+\sum_{j=2}^{m}\left(n_{j}-n_{j-1}\right) \frac{2}{n_{j}-1}\right)^{-1} \\
& =\left(2 m-2 c_{2}^{-1}-\cdots-2 c_{m}^{-1}-c_{2} c_{3} \cdots c_{m}\right)^{-1}
\end{aligned}
$$

That this $v$ gives the optimal $d$ can be shown by comparing $\delta_{v^{\prime}}^{-1}$, for a $v^{\prime}$ with $v_{i}^{\prime} \geq 3$ for some $i$, to $\delta_{v^{\prime \prime}}^{-1}$, where $v^{\prime \prime}$ is obtained from $v^{\prime}$ by letting $v_{i}^{\prime \prime}=v_{i}^{\prime}-1$, and taking $v_{j}^{\prime \prime}=2$ for a $j$ with $v_{j}^{\prime}=1$. Such a $j$ exists because of the condition $n_{m}<2 n_{1}$. We omit further technical details.

Using Proposition 11, it is easy to show that the following holds:

Proposition 6 Let $m \geq 2$ and $2 \leq n_{1} \leq \cdots \leq n_{m}<2 n_{1}$. Then $1 \geq d>\left(2 m\left(1-\sqrt[m]{\frac{1}{2}}\right)\right)^{-1}$.

The lower bound for $d$ is attained when $c_{i}=\sqrt[m]{2}$ for all $i$. We conjecture that this lower bound for $d$ holds in all cases. This conjecture is supported by the results for $m=2$ and $m=3$, see Propositions 2 and 4, respectively.

We remark further that the sequence $\left(2 m\left(1-\sqrt[m]{\frac{1}{2}}\right)\right)^{-1}$ is decreasing in $m$, and converges to $\frac{1}{2 \log 2} \simeq 0.721348$. Hence, if our conjecture is true we will never lose more than $27.87 \%$, with respect to the "restriction free" maximin distance, when nesting the sets $X_{1}, \ldots, X_{m}$. 


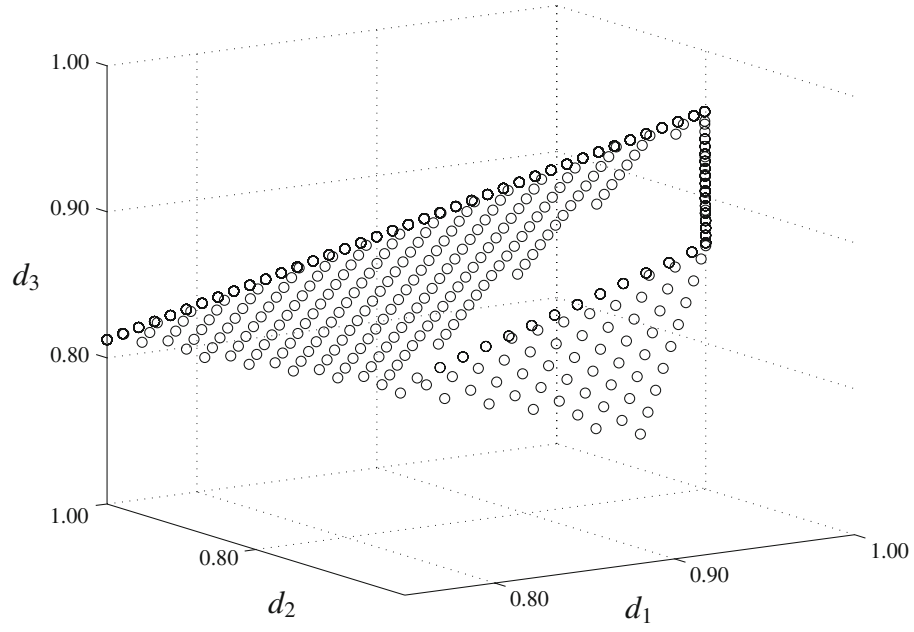

Fig. 7 Dominant combinations for $n_{1}=4, n_{2}=9$, and $n_{3}=14$

For the case $m=4$ we extended the mixed integer linear program for three nested sets, see (8), and found results up to $n_{4}=19$. Unfortunately, as $n_{4}$ gradually increases, the computation time rapidly grows, leading to some instances that take 4 hours to solve. Therefore, we built a heuristic that searches for good nested designs for given $n_{1}, n_{2}, n_{3}, n_{4}$. This heuristic first constructs a nested design for $n_{1}, n_{2}, n_{3}$, see Sect. 3.3, which is conjectured to be an optimal nested design. Then the $n_{4}-n_{3}$ extra points are sequentially added, in the way described in Sect. 3.3. As can be observed from Fig. 8, for $n_{4} \leq 19$ our heuristic often finds the maximin distance (and thus the corresponding nested maximin design), and is not too far off in most other cases. Unfortunately, there is an instance, i.e., $(4,6,9,14)$, for which the maximal distance found by the heuristic has a value that is less than the (conjectured) lower bound in Proposition 6 (the dotted lines in the figure). For this instance the heuristic finds a maximal distance of 0.7796 , which is smaller than the lower bound of 0.7857 and the maximin distance $d(4,6,9,14)=0.7923$.

\section{Conclusions and further research}

In this paper we discussed the construction of nested maximin designs. Such designs play an important role in the design of computer experiments in black-box evaluation and optimization processes. The main reason for using nested designs originates from the area of training and test sets. A nested maximin design consists of two space-filling sets, say, $X_{1}$ and $X_{2} \backslash X_{1}$. The design points in set $X_{1}$ can be used as a training set for fitting a particular metamodel. Set $X_{2} \backslash X_{1}$ can then be used as a test set for validating the obtained metamodel. Note that by using a nested maximin design there is no overlap between the design points in the training set and the test set, while the design points remain to be distributed all over the feasible region. Other reasons for using nested designs are linking parameters and sequential evaluations. Linking parameters occur when several black-box functions share the same input parameters, or when uniformity in parameter settings is needed. We speak of sequential evaluations when an initial set of function evaluations is followed by extra sets of evaluations, as is often the case in practice. 


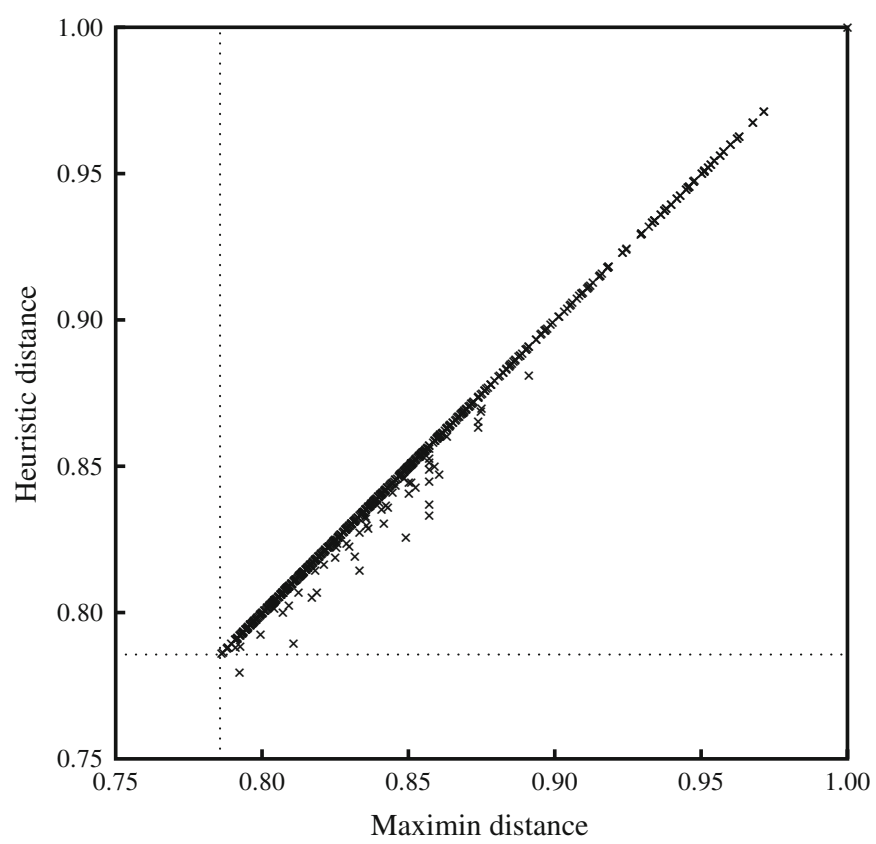

Fig. 8 Maximal distance found by heuristic versus the maximin distance for $n_{4} \leq 19$

Constructing a nested maximin design, which consists of a training set and a test set, or for two black-box functions that share a single linking parameter, or for two-stage sequential evaluations, can be considered as constructing a nested maximin design for two nested sets $X_{1} \subseteq X_{2}=\left\{x_{1}, \ldots, x_{n_{2}}\right\}$, with $x_{j} \in[0,1]$. In this case, the maximin distance equals $d=\left(1+\left\lfloor c_{2}\right\rfloor+\left\lceil c_{2}\right\rceil-c_{2}-\left\lfloor c_{2}\right\rfloor\left\lceil c_{2}\right\rceil \frac{1}{c_{2}}\right)^{-1}$, where $c_{2}=\frac{n_{2}-1}{n_{1}-1}$, and a corresponding nested maximin design is given by (4). It is shown that due to the restriction $X_{1} \subseteq X_{2}$ the resulting designs are at most $14.64 \%$ less space-filling than traditional maximin designs, in case of training and test sets, and linking parameters. For sequential evaluations Fig. 3 shows the net gain of using nested maximin designs. In all cases, it turns out that using nested maximin designs instead of traditional maximin designs is very profitable.

Although we lack an explicit formula for the maximin distance of three nested sets it is proven that this distance is at least 0.807887 . Furthermore, for small instances nested maximin designs can be found by mixed integer linear programming. Fortunately, we developed a fast heuristic that constructs nested designs for larger instances, too. These nested maximin designs can be downloaded from the website http://www.spacefillingdesigns.nl. Based on the obtained results, it is conjectured that this heuristic is optimal, i.e., it will yield a nested maximin design for all instances. An extension of the heuristic to four nested sets often finds nested maximin designs and is not too far off in most other cases.

To investigate the trade-off between nested sets dominant combinations are introduced. In case of two nested sets this relation is linear and is given by (5). For three nested sets the behavior of these dominant combinations is not always that simple, e.g., see Fig. 7. Finally, it is proven that a lower bound on the maximin distance for the general problem of $m$ nested sets is given by $\left(2 m\left(1-\sqrt[m]{\frac{1}{2}}\right)\right)^{-1}$, under the restriction $n_{m}<2 n_{1}$. It is conjectured that this lower bound also holds for all other instances, as is supported by the results for $m=2$ and $m=3$. 
Besides considering the maximin criterion, the concept of nested designs could also be applied to other distance measures, like minimax, IMSE, and maximum entropy. Furthermore, the one-dimensional results in this paper could be extended to more dimensions, thereby constructing multi-dimensional nested maximin designs. Rennen et al. (2009), for example, apply some of the ideas in this paper to the case of such multi-dimensional nested maximin designs.

Open Access This article is distributed under the terms of the Creative Commons Attribution Noncommercial License which permits any noncommercial use, distribution, and reproduction in any medium, provided the original author(s) and source are credited.

\section{References}

Booker, A.J., Dennis, J.E., Frank, P.D., Serafini, D.B., Torczon, V., Trosset, M.W.: A rigorous framework for optimization of expensive functions by surrogates. Struct. Optim. 17(1), 1-13 (1999)

Cherkassky, V., Mulier, F.: Learning from Data: Concepts, Theory, and Methods. Wiley-Interscience Series on Adaptive and Learning Systems for Signal Processing, Communications, and Control. Wiley, Chichester (1998)

den Hertog, D., Stehouwer, H.P.: Optimizing color picture tubes by high-cost nonlinear programming. Euro. J. Oper. Res. 140(2), 197-211 (2002)

Du, D.-Z., Pardalos, P.M. (eds.): Minimax and Applications. Kluwer, Dordrecht (1995)

Gensane, Th.: Dense packings of equal spheres in a cube. Elect. J. Comb. 11, R33 (2004)

Hurrion, R.D.: An example of simulation optimisation using a neural network metamodel: finding the optimum number of kanbans in a manufacturing system. J. Oper. Res. Soc. 48(11), 1105-1112 (1997)

Husslage, B.G.M., van Dam, E.R., Den Hertog, D., Stehouwer, H.P., Stinstra, E.D.: Collaborative metamodeling: coordinating simulation-based product design. Concurr. Eng. Res. Appli. 11(4), 267-278 (2003)

Husslage, B.G.M., Rennen, G., van Dam, E.R., Den Hertog, D.: Space-filling Latin hypercube designs. CentER Discussion Paper 2008-104. Tilburg University, Tilburg (2008)

Jones, D., Schonlau, M., Welch, W.J.: Efficient global optimization of expensive black-box functions. J. Glob. Optim. 13, 455-492 (1998)

Kleijnen, J.P.C., van Beers, W.C.M.: Application-driven sequential designs for simulation experiments: Kriging metamodelling. J. Oper. Res. Soc. 55(8), 876-883 (2004)

Maranas, C., Floudas, C., Pardalos, P.M.: New results in the packing of equal circles in a square. Discret. Math. 142, 287-293 (1995)

Montgomery, D.C.: Design and Analysis of Axperiments. 2nd edn. Wiley, New York (1984)

Morris, M.D., Mitchell, T.J.: Exploratory designs for computer experiments. J. Stat. Plan. Inference 43, 381-402 (1995)

Myers, R.H.: Response surface methodology_current status and future directions. J. Qual. Technol. 31, 30-74 (1999)

Nurmela, K.J., Östergård, P.R.J.: Packing up to 50 equal circles in a square. Discret. Comput. Geom. 18, 111-120 (1997)

Nurmela, K.J., Östergård, P.R.J.: More optimal packings of equal circles in a square. Discret. Comput. Geom. 22, 439-547 (1999)

Pintér J.D.: LGO: A Model Development and Solver System for Continuous Global Optimization. Pintér Consulting Services Inc., Halifax (1995)

Pintér, J.D.: Globally optimized spherical point arrangements: model variants and illustrative results. Ann. Oper. Res. 104, 213-230 (2001)

Pintér, J.D., Kampas, F.J., Castillo, I.: Solving circle packing problems by global optimization: numerical results and industrial applications. Euro. J. Oper. Res. 191, 786-802 (2008)

Rennen, G., Husslage, B.G.M., van Dam, E.R., den Hertog, D.: Nested maximin Latin hypercube designs. Struct. Multidiscip. Optim. (2009) (submitted)

Sacks, J., Welch, W.J., Mitchell, T.J., Wynn, H.P.: Design and analysis of computer experiments. Stat. Sci. 4, 409-435 (1989)

Santner, Th.J., Williams, B.J., Notz, W.I.: The Design and Analysis of Computer Experiments. Springer Series in Statistics. Springer, New York (2003)

Stortelder, W.J.H., de Swart, J.J.B., Pintér, J.D.: Finding elliptic Fekete points sets: two numerical solution approaches. Comput. Appl. Math. 130(1-2), 205-216 (2001)

Su, C.T., Chen, M.C., Chan, H.L.: Applying neural network and scatter search to optimize parameter design with dynamic characteristics. J. Oper. Res. Soc. 56(10), 1132-1140 (2005) 
van Dam, E.R., Husslage, B.G.M., den Hertog, D., Melissen, J.B.M.: Maximin Latin hypercube designs in two dimensions. Oper. Res. 55, 158-169 (2007)

van Dam, E.R., Husslage, B.G.M., Rennen, G.: Bounds for maximin Latin hypercube designs. Oper. Res. 57 (2009) (to appear) 